\title{
Editorial
}

\section{Geometry of Function Spaces}

\author{
T. S. S. R. K. Rao, ${ }^{1}$ Henryk Hudzik, ${ }^{2}$ and Miguel Martin $^{3}$ \\ ${ }^{1}$ Indian Statistical Institute, Bangalore 560059, India \\ ${ }^{2}$ Department of Function Spaces Theory, Faculty of Mathematics and Computer Science, Adam Mickiewicz University, \\ 61-712 Poznań, Poland \\ ${ }^{3}$ Departamento de Análisis Matemático, Facultad de Ciencias, Universidad de Granada, 18071 Granada, Spain
}

Correspondence should be addressed to T. S. S. R. K. Rao; tss@isibang.ac.in

Received 10 December 2013; Accepted 10 December 2013

Copyright ( 92013 T. S. S. R. K. Rao et al. This is an open access article distributed under the Creative Commons Attribution License, which permits unrestricted use, distribution, and reproduction in any medium, provided the original work is properly cited.

In recent years the use of the techniques from the geometric theory of Banach spaces in the study of function spaces has become an important tool and we are happy that this special issue has received the attention of both senior and young researchers in these areas.

We have received papers from a wide cross-section of researchers in this area, covering existence theorems concerning the convolution of functions, distributions, and ultradistributions of Beurling type with supports satisfying suitable compatibility conditions and the Rayleigh equation of two deviating arguments. Application of the Leray-Schauder index theorem and the Leray-Schauder fixed point theorem towards the existence of nontrivial periodic solutions of this type of equations is covered. Also covered is smooth approximation of Lipschitz functions on Finsler manifolds, keeping control on the corresponding Lipschitz constants. An article that extends the usual notion of Köthe dual of a Banach function space to a broad class of Banach lattices is also in this collection.

On abstract Banach space theory, there is an article that studies a weakening of the notion of central subspace of a Banach space, as well as an article that studies notions of generalized approximation properties.

\section{Acknowledgment}

The editors thank all the contributors and colleagues who spared time and were prompt in refereeing work.

\author{
T. S. S. R. K. Rao \\ Henryk Hudzik \\ Miguel Martin
}




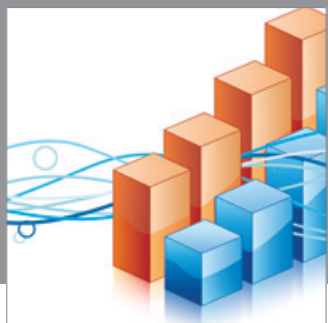

Advances in

Operations Research

mansans

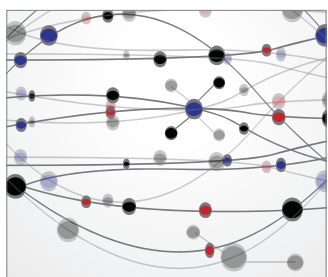

The Scientific World Journal
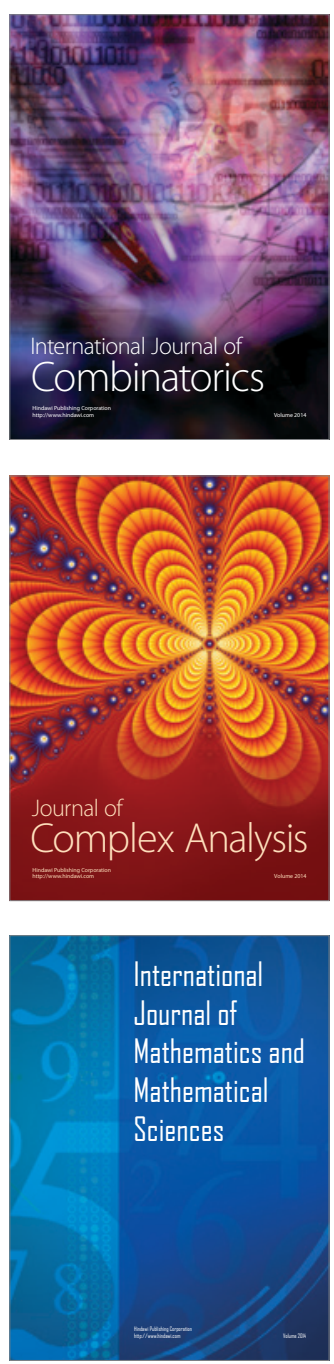
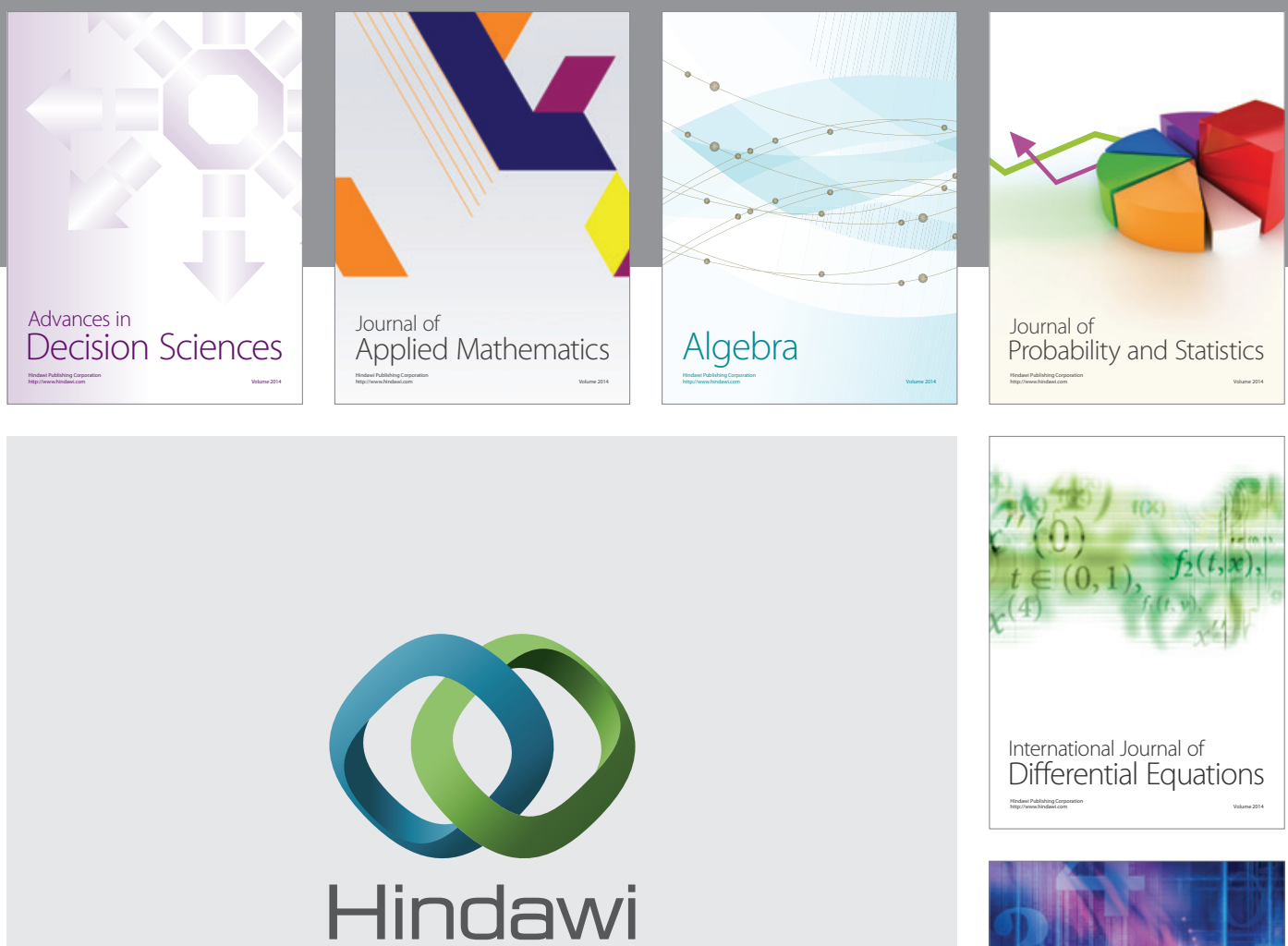

Submit your manuscripts at http://www.hindawi.com
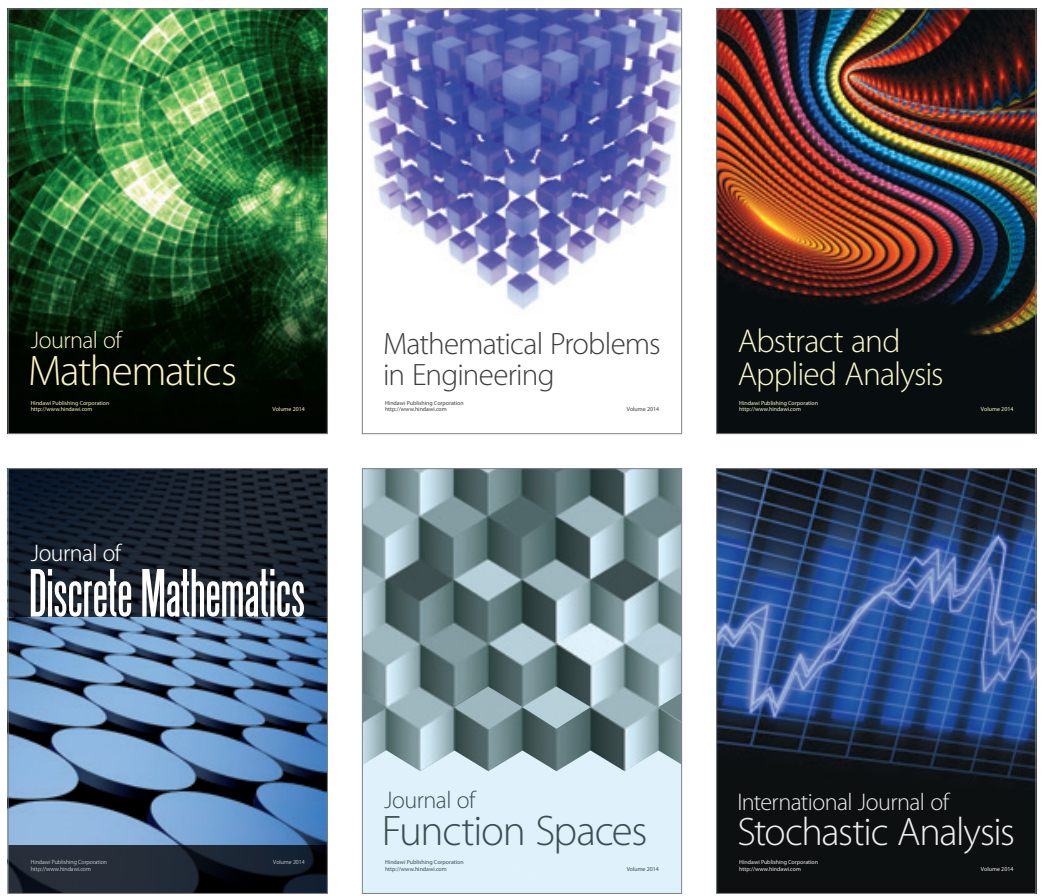

Journal of

Function Spaces

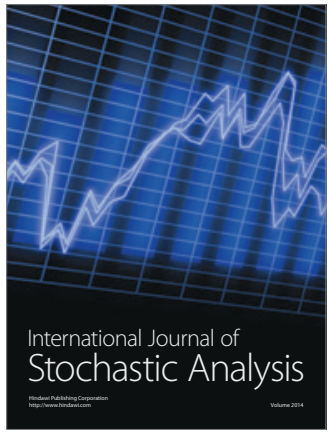

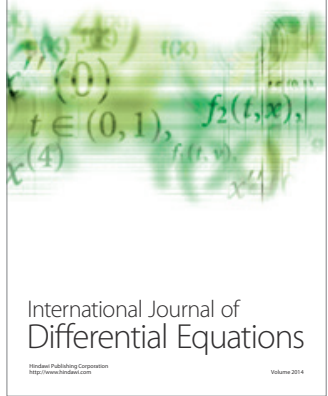
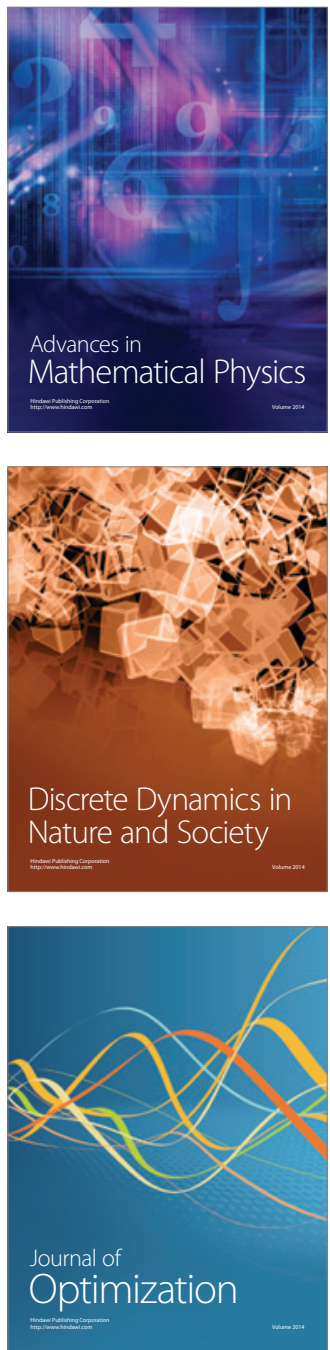